Original Article

\title{
Fluconazole and Ibuprofen Combination: A Potential Treatment for Mucosal Candidiasis
}

Tahere Shamoushaki

(MSc) Department of Biology, Gorgan Branch, Islamic Azad University,

Gorgan, Iran

\section{Leila Fozouni}

(PhD) Department of Biology, Gorgan Branch, Islamic Azad University,

Gorgan, Iran

Corresponding author: Leila Fozouni

Email: lili_kia@yahoo.com

Tel: +989111518674

Address: Department of Biology,

Gorgan Branch, Islamic Azad

University, Gorgan, Iran

Received: 2019/10/14

Revised: 2020/05/3

Accepted: 2020/05/5

\section{cc) (7) (8)}

This work is licensed under a Creative Commons Attribution 4.0 License.

DOI: $10.29252 / \mathrm{mlj} .14 .5 .25$

\begin{abstract}
Background and objectives: The incidence of drug-resistant candidiasis has increased dramatically. This study aimed to evaluate antifungal effects of fluconazole alone and in combination with ibuprofen on isolates from patients with mucosal candidiasis.

Methods: Candida species isolates from 142 patients with suspected mucosal (oral and vaginal) candidiasis were identified by culture on CHROMagar Candida medium and carbohydrate assimilation test using the API 20CAUX kit. Minimum inhibitory concentration (MIC) of fluconazole alone and in combination with ibuprofen was determined by the broth microdilution method.
\end{abstract}

Results: Among isolates, $43.7 \%$ were identified as Candida species (Candida albicans, Candida glabrata, Candidia parapsilosis, Candida tropicalis, Candida guilliermondii and Candida kefyr). The highest rate of fluconazole resistance was observed among C. albicans (50\%) isolates. $\mathrm{MIC}_{90}$ of ibuprofenfluconazole combination against $C$. albicans isolates was 32 $\mu \mathrm{g} / \mathrm{mL}$, which was 8 -fold less than that of fluconazole alone $\left(\mathrm{MIC}_{90}=256 \mu \mathrm{g} / \mathrm{mL}\right) \quad(\mathrm{P}<0.01)$. Moreover, the $\mathrm{MIC}_{90}$ of fluconazole-ibuprofen combination against $C$. parapsilosis isolates was 4-fold less than that of fluconazole alone.

Conclusion: Our results revealed partial fluconazole resistance among Candida isolates from patients with mucosal candidiasis. However, the resistance rate decreased 2.5 fold following treatment with the ibuprofen-fluconazole combination. Therefore, it is recommended to further investigate the therapeutic potential of this drug combination for treatment of fungal infections, such as candidiasis. effect

Keywords: Candida, Fluconazole, Ibuprofen, Antifungal 


\section{INTRODUCTION}

Candidiasis refers to opportunistic fungal infections ranging from simple superficial infections to systemic infections. The infections can appear as acute, sub-acute or chronic in the skin, nails, oral mucosa, vagina, lungs and gastrointestinal tract that may spread to other organs such as kidneys, liver, heart, etc. (1).

Spread of fungal infections and the increasing use of antifungal drugs have dramatically increased the prevalence of resistance to antifungals, such as triazoles. Repeated exposure to antifungal drugs acts as environmental stress that stimulates cellular response to the harmful effects of the drugs, thus allowing sustainable growth of the microorganism. These drug-induced stresses are mediated through signaling pathways (2). Azoles disrupt ergosterol biosynthesis within the membrane by inhibiting fungal cytochrome $\mathrm{P}_{450}$-dependent enzymes, such as lanosterol 14alpha-demethylase, which leads to senescence or apoptosis $(3,4)$.

Ibuprofen [alpha-methyl-4-(2-methyl propyl) benzene acetic acid], also known by the brand names Brufen and Nurofen, is a non-steroidal anti-inflammatory drug (NSAID) used as an antipyretic and analgesic to relieve symptoms of arthritis, dysmenorrhea, gout attacks, sport injuries and inflammatory pain. Similar to other NSAIDs, it inhibits the cyclooxygenase enzyme and counteracts inflammatory precursors by reducing prostaglandin production. The World Health Organization has listed this drug as an essential medicine $(5,6)$.

This study aimed to determine and compare the minimum inhibitory concentration (MIC) of fluconazole alone and combined with ibuprofen against Candida isolates from patients with oral or vaginal candidiasis.

\section{MATERIALS AND METHODS}

In the present cross-sectional study, we collected 142 clinical samples from patients (113 female, age range: 21-75 years) with suspected mucosal candidiasis who were referred to clinical laboratories in Gorgan and Bandar-e Gaz, northern Iran.

In order to identify the isolated microorganisms, swab samples were cultured on Sabouraud dextrose agar (SDA, Merck, Germany) and incubated at $35{ }^{\circ} \mathrm{C}$ for 48 hours.
After direct microscopic examination using $10 \% \mathrm{KOH}$ and subculture on SDA medium, isolates were identified with Gram staining, culture on chromogenic medium (CHROMagar, Hi-Media, India) and carbohydrate assimilation test using the API 20CAUX kit (BioMerieux, France).

Susceptibility to antifungal agents was evaluated using the broth microdilution method according to the CLSI-M27-A3 guidelines (7). Fluconazole stock solution (Gibco, Germany) was diluted with water to obtain a concentration range of $2-1024 \mu \mathrm{g} / \mathrm{mL}$. The prepared fluconazole dilutions were poured into wells of a 96-well microplate containing RPMI 1640 medium (with glutamine, without bicarbonate, and with a $\mathrm{pH}$ indicator) and MOPS buffer (Sigma, USA). Next, a yeast suspension $\left(1 \times 10^{3} \mathrm{CFU} / \mathrm{mL}\right)$ was inoculated into the wells and the plate was incubated at $35{ }^{\circ} \mathrm{C}$ for 48 hours. Finally, MIC values were recorded by reading absorbance at $530 \mathrm{~nm}$ using an ELISA microplate reader (Biotec, Germany). According to the CLSIM27-A3 instructions, Candida strains with MIC values of $\leq 8 \mu \mathrm{g} / \mathrm{mL}, 16-32 \mu \mathrm{g} / \mathrm{mL}$ and $\geq 64 \mu \mathrm{g} / \mathrm{mL}$ were identified as susceptible, susceptible-dose dependent and resistant to fluconazole. In the present study, Candida albicans ATCC90028 was used as the control strain.

In order to prepare combination stock, sufficient amounts of fluconazole and ibuprofen powder were dissolved in water to obtain a concentration of $1024 \mu \mathrm{g} / \mathrm{mL}$. To prepare serial dilutions, $50 \mu \mathrm{L}$ of combination solution were added to the first well of a 96well microplate containing $50 \mu \mathrm{L}$ of RPMI medium. After adding $50 \mu \mathrm{L}$ of yeast suspension $\left(10^{3} \mathrm{CFU} / \mathrm{mL}\right)$ and 48 hours of incubation at $35{ }^{\circ} \mathrm{C}$, MIC values were determined and interpreted.

Data were analyzed using ANOVA and Duncan's multiple range test at a confidence level of 5\%. All statistical analyses were performed using SPSS software and charts were drawn with Excel software.

\section{RESULTS}

Of 142 isolates, $62(43.7 \%)$ were identified as Candida species, $66.1 \%$ of which were isolated from patients with vaginal candidiasis. The majority (79\%) of Candida isolates were C. albicans (Figure 1). 
The results also showed that $50 \%$ of $C$. albicans and $4.8 \%$ of $C$. parapsilosis isolates were resistant to fluconazole. The concentration of ibuprofen combined with fluconazole that inhibited $90 \%$ of $C$. albicans isolates $\left(\mathrm{MIC}_{90}\right)$ was $32 \mu \mathrm{g} / \mathrm{mL}$, which is 8 fold lower than that of fluconazole $\left(\mathrm{MIC}_{90}=\right.$ $256 \mu \mathrm{g} / \mathrm{mL})(\mathrm{P}<0.01)$. This rate was also 4-fold for $C$. parapsilosis and C. glabrata isolates (Table1).
As expected, the combination of ibuprofen and fluconazole reduced the number of resistant Candida isolates and increased the rate of absolute susceptibility. The frequency of fluconazole-resistant $C$. albicans isolates reduced from $63.27 \%$ to $24.49 \%$ when treated with the same dose of ibuprofen-fluconazole combination (Table 2).

Figure 1. Relative frequency of Candida species isolates from patients with mucosal candidiasis

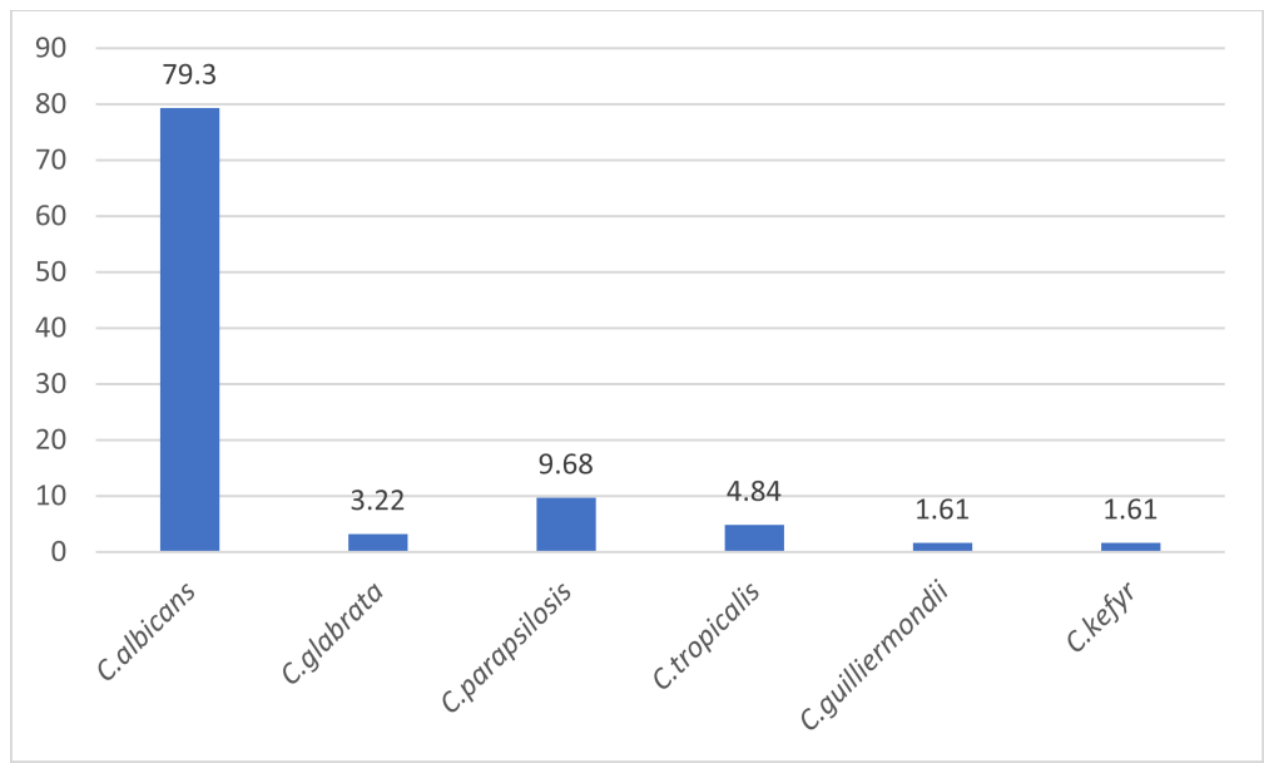

Table1. MICs of ibuprofen and fluconazole combination against Candida species

\begin{tabular}{|c|c|c|c|}
\hline Strains $\left(\mathbf{1} \times \mathbf{1 0}^{\mathbf{3}} \mathbf{c f u} / \mathbf{m l}\right)$ & $\mathbf{M I C}_{\mathbf{9 0}}$ & $\mathbf{M I C}_{\mathbf{5 0}}$ & Comparison \\
\hline C. albicans & $32 \mu \mathrm{g} / \mathrm{ml}$ & $8 \mu \mathrm{g} / \mathrm{ml}$ & $\mathbf{X}^{2}=\mathbf{S}$ \\
\hline C. glabraata & $128 \mu \mathrm{g} / \mathrm{ml}$ & $32 \mu \mathrm{g} / \mathrm{ml}$ & $\mathbf{X}^{2}=\mathbf{S}$ \\
\hline C. parapsilosis & $64 \mu \mathrm{g} / \mathrm{ml}$ & $32 \mu \mathrm{g} / \mathrm{ml}$ & $\mathbf{X}^{2}=\mathbf{N S}$ \\
\hline
\end{tabular}

S: Significant

NS: Not Significant 
Table 2. Frequency of resistance to fluconazole and ibuprofen-fluconazole combination among Candida isolates

\begin{tabular}{|c|c|c|c|}
\hline \multicolumn{2}{|c|}{ Candida species } & \multirow{2}{*}{$\begin{array}{c}\text { Fluconazole } \\
31(66.3 \%)\end{array}$} & \multirow{2}{*}{$\begin{array}{c}\text { Fluconazole + ibuprofen } \\
12(24.5 \%)\end{array}$} \\
\hline \multirow{3}{*}{ C. albicans } & Resistant & & \\
\hline & $\begin{array}{c}\text { Susceptible-dose } \\
\text { dependent }\end{array}$ & $8(16.3 \%)$ & $0(0)$ \\
\hline & Susceptible & $10(20.4 \%)$ & $37(75.5 \%)$ \\
\hline \multirow{3}{*}{ C. glabrata } & Resistant & $1(50 \%)$ & $0(0)$ \\
\hline & $\begin{array}{c}\text { Susceptible-dose } \\
\text { dependent }\end{array}$ & $1(50 \%)$ & $0(0)$ \\
\hline & Susceptible & $0(0)$ & $2(100 \%)$ \\
\hline \multirow{3}{*}{ C. parapsilosis } & Resistant & $3(50 \%)$ & $1(16.7 \%)$ \\
\hline & $\begin{array}{c}\text { Susceptible-dose } \\
\text { dependent }\end{array}$ & $2(33.3 \%)$ & $0(0)$ \\
\hline & Susceptible & $1(16.7 \%)$ & $5(83.3 \%)$ \\
\hline \multirow{3}{*}{ C. tropicalis } & Resistant & $1(33.3 \%)$ & $1(33.3 \%)$ \\
\hline & $\begin{array}{c}\text { Susceptible-dose } \\
\text { dependent }\end{array}$ & $1(33.3 \%)$ & $0(0)$ \\
\hline & Susceptible & $1(33.3 \%)$ & $2(66.7 \%)$ \\
\hline \multirow{3}{*}{ C. quilliermondii } & Resistant & $0(0)$ & $0(0)$ \\
\hline & $\begin{array}{c}\text { Susceptible-dose } \\
\text { dependent }\end{array}$ & $1(100 \%)$ & $0(0)$ \\
\hline & Susceptible & $0(0)$ & $1(100 \%)$ \\
\hline \multirow{3}{*}{ C. kefyr } & Resistant & $0(0)$ & $0(0)$ \\
\hline & $\begin{array}{c}\text { Susceptible-dose } \\
\text { dependent }\end{array}$ & $0(0)$ & $0(0)$ \\
\hline & Susceptible & $1(100 \%)$ & $1(100 \%)$ \\
\hline
\end{tabular}

\section{DISCUSSION}

Prolonged or repeated exposure to antifungal agents is associated with increased emergence of resistant Candida strains $(8,9)$. In this study, $C$. albicans $(79 \%), \quad C$. parapsilosis, C. tropicalis, C. glabrata, $C$. guilliermondii and $C$. kefyr were the most common Candida species isolated from patients with suspected mucosal candidiasis. A previous study in Iran with a larger study population also reported C. albicans (66.5\%), C. parapsilosis $(8.6 \%)$, C. tropicalis $(8.2 \%)$ and $C$. glabrata $(6.1 \%)$ as the most common Candida species isolated from clinical samples (10). However, the mentioned study did not detect $C$. kefyr among the clinical isolates. In 2007, another study in Iran reported $C$. albicans as the primary cause of vaginal candidiasis (11). In 2011, C. albicans was found as the main cause of vaginal candidiasis in women who had been admitted to a hospital in Tonekabon, Iran (12). C. albicans, $C$. dubliniensis and $C$. glabrata were the most frequent causes of vulvovaginitis in women referred to a medical center in Arak, Iran (13). In our study, $C$. albicans was also the most common species isolated from vaginal specimens.
The prevalence of azole resistance has risen dramatically since 1990 (2). In the present study, $50 \%$ of $C$. albicans and $4.8 \%$ of $C$. parapsilosis isolates were resistant to fluconazole. A study in 2004 found no evidence of fluconazole resistance in $C$. albicans or isolates from superinfection with C. glabrata (14).

Our results revealed that a large percentage of Candida isolates exhibit high resistance against fluconazole. Therefore, we evaluated the antifungal efficacy of fluconazole combined with an NSAID, ibuprofen, against these isolates. According to the results, the antibiotic resistance rate decreased to $22.58 \%$ because of the synergistic effect of fluconazole and ibuprofen. In a study on the effectiveness of fluconazole-oral protexin combination for treatment of vaginal candidiasis, there was no synergistic activity observed and both drugs exhibited similar therapeutic efficacy. However, the fluconazole-protexin combination was more effective in relieving symptoms, such as dysuria compared to fluconazole alone (15). Nowrozi and Kazemi reported that ultraviolet radiation could reduce MIC of fluconazole against Candida strains (16). 
In another study, combination of fluconazole with silver nanoparticles showed significantly higher antifungal activity compared to fluconazole alone against isolates from patients with chronic and recurrent candidal vulvovaginitis (17).

\section{CONCLUSION}

Treatment of the isolates with the fluconazole-ibuprofen combination results in a 2.5 -fold decrease in drug resistance rate. Given the synergistic effect of these drugs, it is recommended to further investigate the therapeutic potential of this combination for treatment of fungal infections, such as candidiasis.

\section{ACKNOWLEDGMENTS}

The authors would like to express their gratitude to all colleagues who helped us in this study. The findings of this study have been derived from the MSc thesis of Tahere Shamoushaki, which was carried out in the microbiology laboratory of Islamic Azad University of Gorgan, Iran.

\section{CONFLICT OF INTERESTS}

The authors declare that there is no conflict of interest.

\section{REFERENCES}

1. Maznei kova V. Vaginal candidiasis treatment protocols using miconazole and fluconazde. Akush ginekol (sofila). 2003; 42: 30-40.

2. Matar MJ, Ostrosky-Zeichner L, Paetznick VL, Rodriguez JR, Chen E, Rex JH. Correlation between ETest, Disk Diffusion, and Microdilution methods for antifungal susceptibility testing of fluconazole and voriconazole. Antimicrob Agents Chemother. 2003; 47(5): 1647-51. doi: 10.1128/aac.47.5.1647-1651.2003.

3. Pappas PG, Kauffman CA, Andes D, Benjamin DK Jr, Calandra TF, et al. Clinical practice guidelines for the management of candidiasis: 2009 update by the Infectious Diseases Society of America. Clin Infect Dis. 2009; 48(5): 503-35. doi: 10.1086/596757.

4. Ahmad A, Khan AU. Prevalence of Candida species and potential risk factors for vulvovaginal candidiasis in Aligarh, India. Eur J Obstet Gynecol Reprod Biol. 2009; 144(1): 68-71. doi: 10.1016/j.ejogrb.2008.12.020.

5. Cherif A, Khrouf N, Jabnoun S, Mokrani C, Amara $\mathrm{MB}$, Guellouze $\mathrm{N}$, et al. Randomized pilot study comparing oral ibuprofen with intravenous ibuprofen in very low birth weight infants with patentductus arteriosus. Pediatrics. 2008; 122: 1256-61. doi: 10.1542/peds.2008-1780.

6. World Health Organization. WHO Model List of Essential Medicines. $20^{\text {th }}$ ed. 2017.

7. Clinical and Laboratory Standards Institute (CLSI). Reference method for broth dilution antifungal susceptibility testing of yeasts. $3^{\text {rd }}$ ed. Pennsylvania:Wayne, PA. 2008.

8. Badiee P, Alborzi A, Shakiba E, Ziyaeyan M, Rasuli M. Molecular Identification and in-vitro susceptibility of Candida albicans and C. dubliniensis Isolated from Immunocompromised Patients. Iranian Red Crescent Medical Journal. 2009; 11(4): 391-97.

9. Richter S, Rudolph P. Antifungal Susceptibilities of Candida Species Causing Vulvovaginitis and Epidemiology of Recurrent Cases. Clin Microbiol. 2005; 43(5): 2155-62. doi: 10.1128/JCM.43.5.2155-2162.2005.

10. Mirhendi SH, Makimura K, Shidfar MR Hosseinpour L. Identification and Frequency of Candida Patients isolates by Chromagar candida method. Avicenna Journal of clinical Medicine. 2007; 13(4):11-15.

11. Aghamirian M, keshavarz D, Jahani Hashemi H, sadeghi Qazvini M. Agents associated with candida vulvovaginitis in women referred to health centers in Qazvin. J Qazvin Univ Med Sci. 2007; 11(3): 35-9.

12. Nasrollahi Omran AA, Vakilli L, Jafarpur M. Determination of Vaginal Candidiasis in Women Referred to Shahid Rajaei hospital in Tonekabon(20092010). Medical Laboratory Journal. 2011; 5(1): 1-7.

13. Jamilian M, Mashadi E, Sarmadi F, Banijamali M, Farhadi E, Ghanatpishe E. Frequancy of vulvovaginal Candidiasis species in nonpregnant 15-50 years old women in spring 2005 in Arak. J Arak Uni Med Sci. 2007; 10(2): 7-14.

14. Sobel JD, Wiesenfeld HC, Martens M, Danna P, Hooton TM, Rompalo A, et al. Maintenance Fluconazole Therapy for Recurrent Vulvovaginal Candidiasis. N Engl j Med. 2004; 351: 876-83.

15. Nouraei S, Amir Ali Akbari S, Jorjani M, Alavi Majd HM, frakhteh A, Ghaforian A, et al. Comparison Between the Effect of Fluconazole and Oral Protexin Combination and Fluconazole on the Treatment of Vulvovaginal Candidiasis. J Arak Uni Med Sci. 2013; 16(4):62-73.

16. Nowrozi H, Kazemi A. The Effect of Ultraviolet (UV) Radiation Duration on Drug Susceptibility Testing of Rhizopus spp. to Amphotericin B, Itraconazole and Fluconazole. JSSU. 2014; 22(1): 850-57.

17. Nozari SH, Haydari Kohan F, Ashrafi Khozani M, Ahmadi F, Ghasemi Z, Nami S, et al. Comparison of Antifungal Effect of Fluconazole alone and in Combination with Nanosilver Particles against Candida spesies Isolated from Chronic Candidal Vulvovaginitis. Razi Journal of Medical Science. 2012; 18(93): 8-14.

\section{How to Cite:}

Shamoushaki T, Fozouni L. [Fluconazole and Ibuprofen Combination: A Potential Treatment for Mucosal Candidiasis]. mljgoums. 2020; 14(5): 25-29. DOI: $10.29252 / \mathrm{mlj} .14 .5 .25$ 